\title{
Counting Yang-Mills Instantons by Surface Operator Renormalization Group Flow
}

\author{
Giulio Bonelli, Fran Globlek, and Alessandro Tanzini \\ International School of Advanced Studies (SISSA), via Bonomea 265, 34136 Trieste, Italy and INFN, \\ Sezione di Trieste and Institute for Geometry and Physics, IGAP, via Beirut 2, 34136 Trieste, Italy
}

(Received 1 March 2021; accepted 4 May 2021; published 11 June 2021)

\begin{abstract}
We show that the nonperturbative dynamics of $\mathcal{N}=2$ super-Yang-Mills theories in a self-dual $\Omega$ background and with arbitrary simple gauge group is fully determined by studying renormalization group equations of vacuum expectation values of surface operators generating one-form symmetries. The corresponding system of equations is a nonautonomous Toda chain, the time being the renormalization group scale. We obtain new recurrence relations which provide a systematic algorithm computing multiinstanton corrections from the tree-level one-loop prepotential as the asymptotic boundary condition of the renormalization group equations. We exemplify by computing the $E_{6}$ and $G_{2}$ cases up to two instantons.
\end{abstract}

DOI: 10.1103/PhysRevLett.126.231602

In an ideal world the nonperturbative structure of gauge theories should be computed by quantum equations of motion determined by a symmetry principle. The presence of extended operators generating higher form symmetries in quantum field theory is a powerful tool to concretely realize such a program. A perturbative analysis in a weakly coupled regime, if any, would supply appropriate asymptotic conditions. Extended operators were introduced in the early days of gauge theory in order to describe its different phases [1]. In particular, in the dual superconductor picture of confinement, surface operators play an important role as world-sheet description of the QCD string. Much progress in the description of the gauge theories nonperturbative dynamics has been obtained in the supersymmetric context, thanks to nonrenormalization theorems [2]. The main point is that the effective theory at low energies in the supersymmetric protected sector can be described in terms of a holomorphic function with prescribed singular behavior, called prepotential. The prepotential has been computed from the UV microscopic Lagrangian by introducing an auxiliary gravitational potential, called $\Omega$ background [3]. More recently, surface operators preserving half of the supercharges in supersymmetric theories have attracted new interest [4]. In this Letter, we consider a class of theories where the nonperturbative effects are computed from the properties of surface operators charged under the center of the gauge group. These are $\mathcal{N}=2$ super-Yang-Mills theories in four dimensional self-dual $\Omega$ background, which enjoy a one-form symmetry generated by surface operators [5].

Published by the American Physical Society under the terms of the Creative Commons Attribution 4.0 International license. Further distribution of this work must maintain attribution to the author(s) and the published article's title, journal citation, and DOI. Funded by SCOAP ${ }^{3}$.
We show that the renormalization group equation obeyed by the vacuum expectation value of such surface operators provides a recursion relation which fully determines, from the perturbative one-loop prepotential, all instanton contributions on the self-dual $\Omega$ background or, equivalently, the all-genus topological string amplitudes on the relevant geometric background [6]. Actually, partition functions with surface operators display a very clear resurgent structure led by the summation over the magnetic fluxes [7]. Let us remark that the one-form symmetry, when acting nontrivially, plays an important role in simplifying the analysis of the above renormalization group equations. Being the surface operator described by a supersymmetric gauged linear $\sigma$ model, its dynamics can be described by the relevant $t t^{*}$ equations [8]. Indeed, the system of equations we study is the radial reduction of $t t^{*}$ equations describing complex deformations of a $Z(G)$ singularity, $Z(G)$ being the center of the gauge group. These are the equations of nonautonomous twisted affine Toda chain of type $(\hat{G})^{\vee}$, where $(\hat{G})^{\vee}$ is the Langlands dual of the untwisted affine Kac-Moody algebra $\hat{G}$. Each node of the corresponding affine Dynkin diagram defines a surface operator, the associated $\tau$ function being its vacuum expectation value. The time flow corresponds in the gauge theory to the renormalization group. The resulting recurrence relations constitute a new effective algorithm to determine instanton contributions for all classical groups $G$. Let us remark that the $\tau$ functions we obtain provide the general solution at the canonical rays for the Jimbo-Miwa-Ueno isomonodromic deformation problem $[9,10]$ on the sphere with two irregular punctures for all classical groups, which to the best of our knowledge was not known in the previous literature. The recursion relations we obtain are different from the blowup equations of [11] further elaborated in [12]. Indeed the latter necessarily involve the knowledge of the partition function in different $\Omega$ backgrounds. This makes the 
recursion relations (and the results) coming from blowup equations more involved and difficult to handle. However, we expect a relation between the two approaches to follow from blowup relations in the presence of surface defects. Indeed, the isomonodromic $\tau$ function for the sphere with four regular punctures was obtained in a similar way from $\mathrm{SU}(2)$ gauge theory with $N_{f}=4$ in [13]. In this Letter, we summarize our results and refer to a subsequent longer paper for a fully detailed discussion.

The $\tau$ functions are labeled by the simple roots of the affinization of the Lie algebra of the gauge group $\alpha \in \hat{\Delta}$, namely $\left\{\tau_{\alpha}\right\}_{\alpha \in \hat{\Delta}}$, and satisfy the equations

$$
D^{2}\left(\tau_{\alpha}\right)=-\frac{\left(\boldsymbol{\alpha}^{\vee}, \boldsymbol{\alpha}^{\vee}\right)}{2} t^{1 / h^{\vee}} \prod_{\boldsymbol{\beta} \in \hat{\Delta}, \boldsymbol{\beta} \neq \boldsymbol{\alpha}}\left[\tau_{\boldsymbol{\beta}}\right]^{-\left(\boldsymbol{\beta}, \boldsymbol{\alpha}^{\vee}\right)},
$$

where $t:=(\Lambda / \epsilon)^{2 h^{\vee}}$ and the logarithmic Hirota derivative is given by $D^{2}(f)=f \partial_{\log t}^{2} f-\left(\partial_{\log t} f\right)^{2}$. Given a simple root $\boldsymbol{\alpha}$, its coroot is as usual given by $\boldsymbol{\alpha}^{\vee}=2 \boldsymbol{\alpha} /(\boldsymbol{\alpha}, \boldsymbol{\alpha})$, where $(\cdot, \cdot)$ is the scalar product defined by the affine Cartan matrix. Equation (1) is the deautonomization of the $\tau$ form of the standard Toda integrable system $[14,15]$ governing the classical Seiberg-Witten (SW) theory [16]. The deautonomization is induced by coupling the theory to a self-dual $\Omega$ background $\left(\epsilon_{1}, \epsilon_{2}\right)=(\epsilon,-\epsilon)$ [17]. In the autonomous limit $\epsilon \rightarrow 0, \tau$ functions reduce to $\theta$ functions on the classical SW curve [18], which were used to provide recursion relations on the coefficients of the SW prepotential in [19]. The gauge theory interpretation of these $\tau$ functions is the vacuum expectation value of surface operators associated with the corresponding decomposition of the Lie algebra representation under which these are charged. We expect these equations and their generalizations to describe chiral ring relations in the presence of a surface operator, which deserve further investigation. Higher chiral observables should generate the flows of the full nonautonomous Toda hierarchy. The actual form of Eqs. (1) depends on the Dynkin diagram. For the classical groups $A, B$, and $D$ these reduce to bilinear equations which we solve via general recursion relations. For $C, E, F$, and $G$ the resulting equations are of higher order and we study them case by case. The symmetries of the equations are given by the center of the group $G$, namely

\begin{tabular}{c|c|c|c|c|c|c|c|c}
$\mathfrak{g}$ & $A_{n}$ & $B_{n}$ & $C_{n}$ & $D_{2 n}$ & $D_{2 n+1}$ & $E_{n}$ & $F_{4}$ & $G_{2}$ \\
\hline$Z(G)$ & $\mathbb{Z}_{n+1}$ & $\mathbb{Z}_{2}$ & $\mathbb{Z}_{2}$ & $\mathbb{Z}_{2} \times \mathbb{Z}_{2}$ & $\mathbb{Z}_{4}$ & $\mathbb{Z}_{9-n}$ & 1 & 1
\end{tabular}.

Moreover, the center is isomorphic to the coset of the affine coweight lattice by the affine coroot lattice, and coincides with the automorphism group of the affine Dynkin diagram. By a remark in [20], the coweights, and by extension the lattice cosets, corresponding to these nodes are the minuscule coweights, a representation of $\mathfrak{g}$ being minuscule if all its weights form a single Weyl orbit. This remark will be crucial while solving the $\tau$ system.
The $\tau$ functions corresponding to the affine nodes, that is the ones which can be removed from the Dynkin diagram leaving behind that of an irreducible simple Lie algebra, play a special role. Indeed, these are related to simple surface operators associated with elements of the center $Z(G)$, and are bounded by fractional 't Hooft lines. Such surface operators are the generators of the one-form symmetry of the corresponding gauge theory [5]. Since their magnetic charge is defined modulo the magnetic root lattice, a natural ansatz for their expectation value is

$\tau_{\alpha_{\text {aff }}}\left(\boldsymbol{\sigma}, \boldsymbol{\eta} \mid \kappa_{\mathfrak{g}} t\right)=\sum_{\mathbf{n} \in \mathbf{Q}_{\mathrm{aff}}^{\vee}} e^{2 \pi \sqrt{-1} \eta \cdot \mathbf{n}} t^{(1 / 2)(\boldsymbol{\sigma}+\mathbf{n})^{2}} B(\boldsymbol{\sigma}+\mathbf{n} \mid \mathbf{t})$,

where $B(\boldsymbol{\sigma} \mid t)=B_{0}(\boldsymbol{\sigma}) \sum_{i \geq 0} t^{i} Z_{i}(\boldsymbol{\sigma})$ with $Z_{0}(\boldsymbol{\sigma}) \equiv 1$ and $Q_{\text {aff }}^{\vee}=\lambda_{\text {aff }}^{\vee}+Q^{\vee}, Q^{\vee}$ being the coroot lattice equipped with the canonical inner product normalized such that the norm of the short coroots is 2 , and $\left(\lambda_{\text {aff }}^{\vee}, \boldsymbol{\alpha}\right)=\delta_{\text {aaff, } \boldsymbol{\alpha}}$ for any nonextended simple root $\boldsymbol{\alpha}$. The constant $\kappa_{\mathfrak{g}}=\left(n_{\mathfrak{g}}\right)^{r_{\mathfrak{g}, s} \text {, }}$ where $n_{\mathfrak{g}}$ is the ratio of the squares of long vs short roots and $r_{\mathfrak{g}, s}$ is the number of short simple roots. For simply laced, all roots are long and $\kappa_{\mathfrak{g}}=1$.

We will now show how the term $t^{(1 / 2) \sigma^{2}} B(\boldsymbol{\sigma} \mid t)$ in (2) is the full Nekrasov partition function in the self-dual $\Omega$ background upon the identification $\boldsymbol{\sigma}=\mathbf{a} / \epsilon$, where $\mathbf{a}$ is the Cartan parameter. In the $A_{n}$ case, (2) is known as the Kiev ansatz. In the $A_{1}$ case, it was used to give the general solution of Painlevé $\mathrm{III}_{3}$ equation in [21] and further analyzed in [22].

Let us remark that the $\tau$ function (2) displays a clear resurgent structure, with "instantons" given by the magnetic fluxes in the lattice summed with "resurgent" coefficients $B(\boldsymbol{\sigma} \mid t)$ and trans-series parameter $e^{2 \pi \sqrt{-1}} \boldsymbol{\eta}$; see [23] for a similar analysis in the Painlevé $\mathrm{III}_{3}$ case.

The ansatz (2) is consistent with Eqs. (1). Indeed, after eliminating the $\tau$ functions associated with the nonaffine nodes, the resulting equation is bilinear and therefore the ansatz (2) reduces to a set of recursion relations for the coefficients $Z_{i}(\boldsymbol{\sigma})$. The variables $\boldsymbol{\eta}, \boldsymbol{\sigma} \in Q^{\vee}$ are the integration constants of the second order differential equations (1) and correspond to the initial position and velocity of the deautonomized Toda particle.

Let us set more precisely the boundary conditions which we impose to the solutions of Eqs. (1). We consider the asymptotic behavior of the solutions at $t \rightarrow 0$ and $\sigma \rightarrow \infty$ as

$$
\log \left(B_{0}\right) \sim-\frac{1}{4} \sum_{\mathbf{r} \in \mathbf{R}}(\mathbf{r} \cdot \boldsymbol{\sigma})^{\mathbf{2}} \log (\mathbf{r} \cdot \boldsymbol{\sigma})^{\mathbf{2}}
$$

up to quadratic and log terms [24]. We will show that the solution of (1) which satisfies the above asymptotic condition is such that

$$
B_{0}(\boldsymbol{\sigma})=\mathcal{Z}_{1-\text { loop }}(\boldsymbol{\sigma}) \equiv \prod_{\mathbf{r} \in \mathbf{R}} \frac{1}{G(1+\mathbf{r} \cdot \boldsymbol{\sigma})},
$$


where $G(z)$ is the Barnes $G$ function and $R$ is the adjoint representation of the group $G$. The expansion of the above function matches the one-loop gauge theory result upon the appropriate identification of the log-branch. This reads, in the gauge theory variables, as $\ln [\sqrt{-1} \mathbf{r} \cdot \mathbf{a} / \Lambda] \in \mathbb{R}$ and in the $A_{n}$ case matches the canonical Stokes rays obtained in [25].

Let us first focus on the $A_{n}$ case whose affine Dynkin diagram is

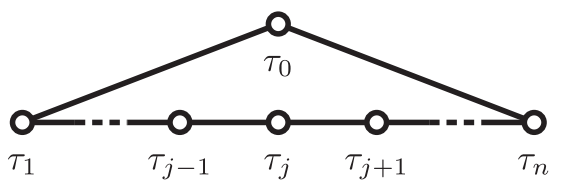

The root lattice is $Q=\left\{\sum_{i=1}^{n+1} c_{i} e_{i} \mid \sum_{i=1}^{n+1} c_{i}=0\right\}$, and all the fundamental weights are minuscule, namely

$$
\lambda_{i}=\left(1^{i}, 0^{n+1-i}\right)-\frac{i}{n+1}\left(1^{n+1}\right),
$$

where $\left(1^{p}, 0^{n+1-p}\right)$ stands for a vector whose first $p$ entries are 1 and the remaining entries vanish. We label the $\tau$ functions as $\tau_{\alpha_{j}} \equiv \tau_{j}$. The $\tau$ system is given by the closed chain of differential equations

$$
D^{2}\left(\tau_{j}\right)=-t^{1 /(n+1)} \tau_{j-1} \tau_{j+1},
$$

with $\tau_{j}=\tau_{n+1+j}$. Since all the nodes in this case are affine we can use the Kiev ansatz (2). Then, all the $\tau$ functions are determined by $\tau_{0}$ as $\tau_{j}=\left.\tau_{0}\right|_{Q \mapsto Q_{j}}$. It is therefore enough to solve (5) corresponding to $j=0$. Here and in the following we use the notation $f(y \pm x) \equiv f(y+x) f(y-x)$. The ansatz (2) for $\tau_{0}$ reads

$\tau_{0}(\boldsymbol{\sigma}, \boldsymbol{\eta} \mid t)=\sum_{\mathbf{n} \in Q, i \geq 0} e^{2 \pi \sqrt{-1} \mathbf{n} \cdot \boldsymbol{\eta}} t^{(1 / 2)(\boldsymbol{\sigma}+\mathbf{n})^{2}+i} B_{0}(\boldsymbol{\sigma}+\mathbf{n}) \mathbf{Z}_{i}(\boldsymbol{\sigma}+\mathbf{n})$

and by inserting it into (5) one gets after some simplifications

$$
\begin{aligned}
& \sum_{\substack{\mathbf{n}_{1}, \mathbf{n}_{2} \in \mathbf{Q} \\
i_{1}, i_{2} \geq 0}} e^{2 \pi \sqrt{-1}\left(\mathbf{n}_{\mathbf{1}}+\mathbf{n}_{\mathbf{2}}\right) \cdot \boldsymbol{\eta}} t^{(1 / 2) \mathbf{n}_{1}^{2}+(\mathbf{1} / \mathbf{2}) \mathbf{n}_{2}^{2}+i_{1}+i_{2}+\boldsymbol{\sigma} \cdot\left(\mathbf{n}_{1}+\mathbf{n}_{2}\right)} \\
& \times\left(\frac{1}{2} \mathbf{n}_{1}^{2}-\frac{\mathbf{1}}{\mathbf{2}} \mathbf{n}_{2}^{\mathbf{2}}+i_{1}-i_{\mathbf{2}}+\boldsymbol{\sigma} \cdot\left(\mathbf{n}_{\mathbf{1}}-\mathbf{n}_{2}\right)\right)^{2} \\
& \times B_{0}\left(\boldsymbol{\sigma}+\mathbf{n}_{1}\right) \mathbf{B}_{0}\left(\boldsymbol{\sigma}+\mathbf{n}_{\mathbf{2}}\right) \mathbf{Z}_{i_{1}}\left(\boldsymbol{\sigma}+\mathbf{n}_{\mathbf{1}}\right) \mathbf{Z}_{i_{2}}\left(\boldsymbol{\sigma}+\mathbf{n}_{\mathbf{2}}\right) \\
& =-\sum_{\substack{\mathbf{m}_{1}, \mathbf{m}_{2} \in \mathbf{Q} \\
j_{1}, j_{2} \geq 0}} t^{1+(1 / 2) \mathbf{m}_{1}^{2}+(\mathbf{1} / \mathbf{2}) \mathbf{m}_{2}^{2}+\mathbf{e}_{1} \cdot\left(\mathbf{m}_{1}-\mathbf{m}_{2}\right)+\mathbf{j}_{1}+\mathbf{j}_{2}+\boldsymbol{\sigma} \cdot\left(\mathbf{m}_{1}+\mathbf{m}_{2}\right)} \\
& \times e^{2 \pi \sqrt{-1}\left(\mathbf{m}_{\mathbf{1}}+\mathbf{m}_{2}\right) \cdot \boldsymbol{\eta}} B_{0}\left(\boldsymbol{\sigma}+\mathbf{m}_{\mathbf{1}}+\mathbf{e}_{\mathbf{1}}\right) \mathbf{B}_{\mathbf{0}}\left(\boldsymbol{\sigma}+\mathbf{m}_{2}-\mathbf{e}_{\mathbf{1}}\right) \\
& \times Z_{j_{1}}\left(\boldsymbol{\sigma}+\mathbf{m}_{1}+\mathbf{e}_{\mathbf{1}}\right) Z_{j_{2}}\left(\boldsymbol{\sigma}+\mathbf{m}_{2}-\mathbf{e}_{\mathbf{1}}\right) \text {. }
\end{aligned}
$$

Now we simply equate the exponents. To fix $B_{0}(\boldsymbol{\sigma})$, we look at the lowest order in $t$. This produces a quadratic constraint and $n+1$ linear constraints on the root lattice variables $\left(\mathbf{n}_{\mathbf{1}}, \mathbf{n}_{\mathbf{2}}\right)$ and $\left(\mathbf{m}_{\mathbf{1}}, \mathbf{m}_{\mathbf{2}}\right)$. Let us fix $p, q \in\{0, \ldots n+1\}, p \neq q$. Up to Weyl reflections, the only solution to the above mentioned constraints is given by $\mathbf{n}_{\mathbf{1}}=\mathbf{e}_{\mathbf{p}}-\mathbf{e}_{\mathbf{q}}, \quad \mathbf{n}_{\mathbf{2}}=\mathbf{0}$, and $\mathbf{m}_{1}=\mathbf{e}_{\mathbf{p}}-\mathbf{e}_{\mathbf{1}}$, $\mathbf{m}_{\mathbf{2}}=-\mathbf{e}_{\mathbf{q}}+\mathbf{e}_{\mathbf{1}}$, leading to

$$
\begin{aligned}
& {\left[1+\left(e_{p}-e_{q}\right) \cdot \boldsymbol{\sigma}\right]^{2} B_{0}\left(\boldsymbol{\sigma}+e_{p}-e_{q}\right) B_{0}(\boldsymbol{\sigma})} \\
& \quad=-B_{0}\left(\boldsymbol{\sigma}+e_{p}\right) B_{0}\left(\boldsymbol{\sigma}-e_{q}\right) .
\end{aligned}
$$

This is solved by (4) up to a function periodic on the root lattice, which is set to one by the asymptotic condition (3). The higher order terms in (6) provide the recursion relations

$$
\begin{aligned}
k^{2} Z_{k}(\boldsymbol{\sigma})= & -\sum_{\substack{\mathbf{n}^{2}+\mathbf{j}_{1}+\mathbf{j}_{2}=\mathbf{k} \\
\mathbf{n} \in \mathbf{e}_{1}++\mathbf{j}_{1,2}<\mathbf{k}}} \frac{B_{0}(\boldsymbol{\sigma} \pm \mathbf{n})}{B_{0}(\boldsymbol{\sigma})^{2}} \times Z_{j_{2}}(\boldsymbol{\sigma}-\mathbf{n}) \mathbf{Z}_{\mathbf{j}_{1}}(\boldsymbol{\sigma}+\mathbf{n}) \\
& +\sum_{\substack{\mathbf{n}^{2}+i_{1}+i_{2}=\mathbf{k} \\
\mathbf{n} \in \mathbf{Q}, i_{1}, 2<\mathbf{k}}}\left(i_{1}-i_{2}+\mathbf{2 n} \cdot \boldsymbol{\sigma}\right)^{\mathbf{2}} \\
& \times \frac{B_{0}(\boldsymbol{\sigma} \pm \mathbf{n})}{B_{0}(\boldsymbol{\sigma})^{2}} Z_{i_{1}}(\boldsymbol{\sigma}+\mathbf{n}) \mathbf{Z}_{i_{2}}(\boldsymbol{\sigma}-\mathbf{n})
\end{aligned}
$$

where $B_{0}(\boldsymbol{\sigma})$ is given by (4). For $k=1$ we easily obtain

$$
Z_{1}(\boldsymbol{\sigma})=-\sum_{i=1}^{n+1} \frac{B_{0}\left(\boldsymbol{\sigma} \pm e_{i}\right)}{B_{0}(\boldsymbol{\sigma})^{2}}=(-1)^{n+1} \sum_{i=1}^{n+1} \frac{1}{\prod_{j \neq i}\left(\sigma_{i}-\sigma_{j}\right)^{2}}
$$

and, upon abbreviating $\sigma_{i j}=\sigma_{i}-\sigma_{j}$, the next term

$$
\begin{aligned}
Z_{2}(\boldsymbol{\sigma})= & -\frac{1}{4} \sum_{i=1}^{n+1} \frac{B_{0}\left(\boldsymbol{\sigma} \pm e_{i}\right)}{B_{0}(\boldsymbol{\sigma})^{2}}\left[Z_{1}\left(\boldsymbol{\sigma}+e_{i}\right)+Z_{1}\left(\boldsymbol{\sigma}-e_{i}\right)\right] \\
& +\sum_{i<j}^{n+1}\left(\sigma_{i}-\sigma_{j}\right)^{2} \frac{B_{0}\left[\boldsymbol{\sigma} \pm\left(e_{i}-e_{j}\right)\right]}{B_{0}(\boldsymbol{\sigma})^{2}}
\end{aligned}
$$

The above coincide with one and two instanton contributions to the $\mathrm{SU}(n+1)$ Nekrasov partition function as computed from supersymmetric localization $[3,26]$. Let us remark that the use of the $\tau$ system (5) provides a completely independent tool to compute all instanton corrections just starting from the asymptotic behavior (3). This procedure extends to all classical groups.

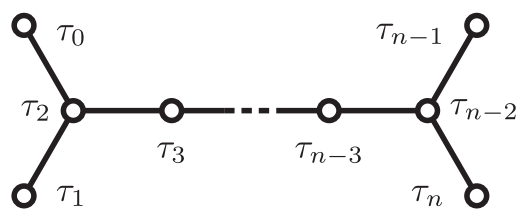


$D_{n}$ is a simply laced root system, with the checkerboard lattice $Q=Q^{\vee}=\left\{\sum_{i=1}^{n} c_{i} e_{i} \mid \sum_{i=1}^{n} c_{i} \in 2 \mathbb{Z}\right\}$. We consider $n>4$. It has four minuscule weights, $\lambda_{0}=\left(0^{n}\right)$, $\lambda_{1}=\left(1,0^{n-1}\right), \quad \lambda_{n-1}=\left[\left(\frac{1}{2}\right)^{n-1},-\frac{1}{2}\right], \quad \lambda_{n}=\left[\left(\frac{1}{2}\right)^{n-1},+\frac{1}{2}\right]$. These correspond to the "legs" of the affine diagram. Whichever rank we consider, we always have the consistency conditions

$$
D^{2}\left(\tau_{0}\right)=D^{2}\left(\tau_{1}\right), \quad D^{2}\left(\tau_{n-1}\right)=D^{2}\left(\tau_{n}\right)
$$

which are also equal if $n=4$, due to the enhanced symmetry of $D_{4}$.

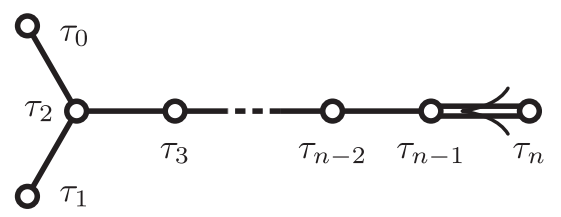

$B_{n}$ is nonsimply laced. The coroot lattice is the checkerboard lattice $Q^{\vee}=\left\{\sum_{i=1}^{n} c_{i} e_{i} \mid \sum_{i=1}^{n} c_{i} \in 2 \mathbb{Z}\right\}$, and the two minuscule weights are $\lambda_{0}^{\vee}=\left(0^{n}\right)$ and $\lambda_{1}^{\vee}=\left(1,0^{n-1}\right)$, corresponding to the "antennas" of the diagram. The $\tau$ system coincides with that of $D_{n+1}$, with the modification that (i) there is no $\tau_{n+1}$ and (ii) that

$$
D^{2}\left(\tau_{n-1}\right)=-2 t^{1 /(2 n-1)} \tau_{n-2} \tau_{n}, \quad D^{2}\left(\tau_{n}\right)=-t^{1 /(2 n-1)} \tau_{n-1}^{2} .
$$

For $n \geq 3$, the analysis proceeds as for $D_{n}$ except we may only use the left antennas and consider the first equation in (8). Therefore, we have a unified approach for both $D_{n}$ and $B_{n}$. Explicitly, inserting (2) and $\tau_{1}(\boldsymbol{\sigma} \mid t)=\tau_{0}\left(\boldsymbol{\sigma}+\lambda_{1} \mid t\right)$ into the first of (8) we get after some simplification a formula analogous to (6) leading to quadratic and linear constraints on the lattice labels. By repeating the analysis similarly to the previous case, the equation, analogous to (7), fixing $B_{0}$ is

$$
\begin{aligned}
& {\left[1+\left(e_{p}+e_{q}\right) \cdot \boldsymbol{\sigma}\right]^{2} B_{0}(\boldsymbol{\sigma}) B_{0}\left(\boldsymbol{\sigma}+e_{p}+e_{q}\right)} \\
& \quad=\left[\left(e_{p}-e_{q}\right) \cdot \boldsymbol{\sigma}\right]^{2} B_{0}\left(\boldsymbol{\sigma}+e_{p}\right) B_{0}\left(\boldsymbol{\sigma}+e_{q}\right) .
\end{aligned}
$$

The two cases are distinguished by the corresponding different asymptotic conditions (3). Indeed, we have

$$
\begin{aligned}
B_{0}^{\left[D_{n}\right]}(\boldsymbol{\sigma}) & =\prod_{i<j}^{n} \frac{1}{G\left(1 \pm \sigma_{i} \pm \sigma_{j}\right)}, \\
B_{0}^{\left[B_{n}\right]}(\boldsymbol{\sigma}) & =\left(\prod_{k=1}^{n} \frac{1}{G\left(1 \pm \sigma_{k}\right)}\right) B_{0}^{\left[D_{n}\right]}(\boldsymbol{\sigma}) .
\end{aligned}
$$

Also, the recursion relations are the same, upon using the appropriate root systems $R$ :

$$
\begin{aligned}
k^{2} Z_{k}(\boldsymbol{\sigma})= & \sum_{\substack{\left(\mathbf{n}-\lambda_{1}\right)^{2}+\mathbf{j}_{1}+\mathbf{j}_{2}=\mathbf{k} \\
\mathbf{n} \in \mathbf{1}_{1}+\mathbf{j}_{\mathbf{j}_{1}, 2}<\mathbf{k}}} Z_{j_{1}}(\boldsymbol{\sigma}+\mathbf{n}) \mathbf{Z}_{\mathbf{j}_{2}}(\boldsymbol{\sigma}-\mathbf{n}) \\
& \times\left(j_{1}-j_{2}+2 \mathbf{n} \cdot \boldsymbol{\sigma}\right)^{2} \frac{B_{0}(\boldsymbol{\sigma} \pm \mathbf{n})}{B_{0}(\boldsymbol{\sigma})^{2}}-\sum_{\substack{\mathbf{n}^{2}+i_{1}+i_{2}=\mathbf{k} \\
\mathbf{n} \in \mathbf{Q}, i_{1}<\mathbf{k}}} Z_{j_{1}}(\boldsymbol{\sigma}+\mathbf{n}) \\
& \times Z_{j_{2}}(\boldsymbol{\sigma}-\mathbf{n})\left(i_{1}-i_{2}+2 \mathbf{n} \cdot \boldsymbol{\sigma}\right)^{2} \frac{\mathbf{B}_{0}(\boldsymbol{\sigma} \pm \mathbf{n})}{\mathbf{B}_{0}(\boldsymbol{\sigma})^{2}}
\end{aligned}
$$

This result is in line with the contour integral formulas for the relevant Nekrasov partition functions. Indeed the poles in the $D_{n}$ and $B_{n}$ cases are the same, with different residues. From the above recursion relation we can compute the 1-instanton terms

$Z_{1}(\boldsymbol{\sigma})=\sum_{k=1}^{n} 4 \sigma_{k}^{2} \frac{B_{0}\left(\boldsymbol{\sigma} \pm e_{k}\right)}{B_{0}(\boldsymbol{\sigma})^{2}}= \begin{cases}\sum_{k=1}^{n} \frac{-4}{\prod_{j \neq k}\left(\sigma_{k}^{2}-\sigma_{j}^{2}\right)^{2}}, & B_{n} \\ \sum_{k=1}^{n} \frac{4 \sigma_{k}^{2}}{\prod_{j \neq k}\left(\sigma_{k}^{2}-\sigma_{j}^{2}\right)^{2}}, & D_{n}\end{cases}$

and the two instantons

$$
\begin{aligned}
Z_{2}(\boldsymbol{\sigma})= & \sum_{\boldsymbol{\alpha} \in Q^{\vee}, \boldsymbol{\alpha}^{2}=2} \frac{-1}{(\boldsymbol{\alpha} \cdot \boldsymbol{\sigma})^{2}\left[(\boldsymbol{\alpha} \cdot \boldsymbol{\sigma})^{2}-1\right]^{2} \prod_{\boldsymbol{\beta} \cdot \boldsymbol{\alpha}=1}(\boldsymbol{\beta} \cdot \boldsymbol{\sigma})^{2}} \\
& +\sum_{k=1}^{n} \frac{Z_{1}\left(\boldsymbol{\sigma}+e_{k}\right)\left(\sigma_{k}+\frac{1}{2}\right)^{2}+Z_{1}\left(\boldsymbol{\sigma}-e_{k}\right)\left(\sigma_{k}-\frac{1}{2}\right)^{2}}{\prod_{\boldsymbol{\beta} \cdot e_{k}= \pm 1}(\boldsymbol{\beta} \cdot \boldsymbol{\sigma})},
\end{aligned}
$$

and so on. These are easily compared to [27].

We now turn to the analysis of the other classical groups, which is more involved. Indeed, the $\tau$ system reduces to higher order equations which produce more complicated recurrence relations to be solved by a case by case analysis. We performed explicit checks for $C_{3}, C_{4}$, and $C_{5}$ up to two instantons again in agreement with [27].

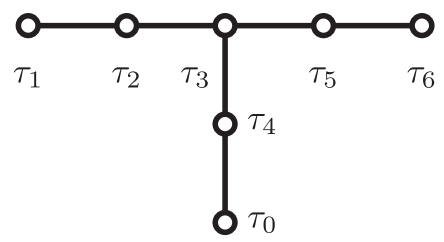

For the exceptional group $E_{6}$ we obtain the system

$$
\tau_{6} D^{4}\left(\tau_{0}\right)=\tau_{0} D^{4}\left(\tau_{6}\right)
$$

where we used the notation $D^{2 n}:=D^{2} \circ D^{2 n-2}$. The equations which specify $B_{0}$ can be written as follows. Choose the minuscule weight to be $\lambda=\left[0^{5},\left(-\frac{2}{3}\right)^{3}\right]$. Let $p_{1}, \ldots, p_{5}$ be a permutation of $\{1, \ldots, 5\}$ and let $\delta:=\left[\left(\frac{1}{2}\right)^{8}\right]$. Then one gets from the lowest order in (10) 


$$
\begin{aligned}
(1+ & \left.\sigma_{p_{1}}+\sigma_{p_{2}}\right)^{2}\left(1+\sigma_{p_{1}}+\sigma_{p_{3}}\right)^{2}\left(\sigma_{p_{2}}-\sigma_{p_{3}}\right)^{2} \\
& \times B_{0}(\boldsymbol{\sigma}) B_{0}\left(\boldsymbol{\sigma}+e_{p_{1}}+e_{p_{2}}\right) B_{0}\left(\boldsymbol{\sigma}+e_{p_{1}}+e_{p_{3}}\right) \\
= & {\left[\left(\boldsymbol{\delta}-e_{p_{2}}-e_{p_{3}}\right) \cdot \boldsymbol{\sigma}\right]^{2}\left[\left(\boldsymbol{\delta}-e_{p_{2}}-e_{p_{3}}-e_{p_{4}}-e_{p_{5}}\right) \cdot \boldsymbol{\sigma}\right]^{2} } \\
& \times\left(\sigma_{p_{4}}+\sigma_{p_{5}}\right)^{2} B_{0}(\boldsymbol{\sigma}+\boldsymbol{\delta}+\lambda) \\
& \times B_{0}\left(\boldsymbol{\sigma}+\boldsymbol{\delta}+\lambda-e_{p_{4}}-e_{p_{5}}\right) B_{0}\left(\boldsymbol{\sigma}+e_{p_{1}}-\lambda / 2\right) .
\end{aligned}
$$

The solution satisfying the asymptotic behavior (3) is $B_{0}^{\left[E_{6}\right]}=$

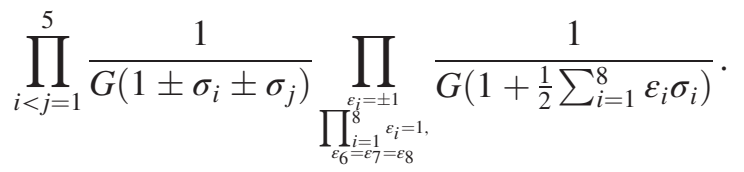

We also solved the recurrence relation arising from (10) up to two instantons. For one instanton, our results agree with the ones of [28], while the two instantons result is a too huge of a formula to be reported here. We remark that (10) represents a completely novel way of obtaining equivariant volumes of instanton moduli spaces for exceptional groups.

Unimodular algebras $G_{2}, F_{4}, E_{8}$ have no outer automorphisms and consequently all the $\tau$ functions associated with different nodes are independent. Indeed in this case there is not a one-form symmetry and therefore the equations on the $\tau$ function associated with the affine node turn out to be more difficult to solve. Let us display them for the $G_{2}$ case.

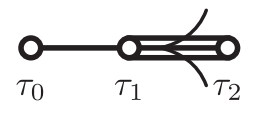

In the normalization where its longest root has length 2 , the $G_{2}$ coroot lattice is the span $Q^{\vee}=\mathbb{Z}(1 / \sqrt{3})(-2,1,1) \oplus$ $\mathbb{Z} \sqrt{3}(1,-1,0)$. We introduce $\boldsymbol{\sigma}=\left(\sigma_{1}, \sigma_{2}, \sigma_{3}\right)$ but all expressions should be restricted to $\sigma_{1}+\sigma_{2}+\sigma_{3}=0$. By eliminating $\tau_{1}$ and $\tau_{2}$, the $\tau$ system reduces to the single equation

$$
D^{2}\left[\tau_{0}^{-1} D^{4}\left(\tau_{0}\right)\right]=3 t\left[D^{2}\left(\tau_{0}\right)\right]^{3} .
$$

The operator on the left-hand side of (11) turns out to factorize as $D^{2}\left[\tau_{0}^{-1} D^{4}\left(\tau_{0}\right)\right]=\tilde{D}^{4}\left(\tau_{0}\right) \cdot D^{2}\left(\tau_{0}\right)$, where $\tilde{D}^{4}\left(\tau_{0}\right)$ is a fourth order operator in $\tau_{0}$ and its derivatives. The trivial solution of $D^{2}\left(\tau_{0}\right)=0$ is $\tau_{0}=a t^{b}$ which we discard being incompatible with (2). In the remainder we insert

$$
\tau_{0}(\boldsymbol{\sigma}, \boldsymbol{\eta} \mid t)=\sum_{\mathbf{n} \in \mathbf{Q}^{\vee}} e^{2 \pi \sqrt{-1} \boldsymbol{\eta} \cdot \mathbf{n}}(-t / 3)^{(1 / 2)(\boldsymbol{\sigma}+\mathbf{n})^{2}} B_{0}\left(\boldsymbol{\sigma}+\mathbf{n} \mid+\frac{t}{3}\right)
$$

and obtain, after a rescaling $t \mapsto 3 t$,

$$
\begin{gathered}
\sum_{\substack{\left.\left\{\mathbf{n}_{\mathbf{k}}\right\} \in \mathbb{V}^{\vee} \\
i_{k}\right\} \in \mathbb{N}}} \prod_{k=1}^{4} e^{2 \pi \sqrt{-1} \eta \cdot \mathbf{n}_{\mathbf{k}}} t^{(1 / 2)\left(\boldsymbol{\sigma}+\mathbf{n}_{\mathbf{k}}\right)^{2}+i_{\mathbf{k}}} B_{0}\left(\boldsymbol{\sigma}+\mathbf{n}_{\mathbf{k}}\right) \mathbf{Z}_{i_{\mathbf{k}}}\left(\boldsymbol{\sigma}+\mathbf{n}_{\mathbf{k}}\right) \\
\quad\left[\frac{1}{4 !} \prod_{k_{1}<k_{2}}\left(\frac{1}{2} \mathbf{n}_{\mathbf{k}_{\mathbf{1}}}^{2}+i_{\mathbf{k}_{\mathbf{1}}}-\frac{1}{2} \mathbf{n}_{\mathbf{k}_{\mathbf{2}}}^{\mathbf{2}}-i_{\mathbf{k}_{\mathbf{2}}}+\left(\mathbf{n}_{\mathbf{k}_{\mathbf{1}}}-\mathbf{n}_{\mathbf{k}_{\mathbf{2}}}\right) \cdot \boldsymbol{\sigma}\right)^{\mathbf{2}}\right. \\
\quad-\frac{9}{4}\left(\frac{1}{2} \mathbf{n}_{\mathbf{1}}^{\mathbf{2}}+i_{\mathbf{1}}-\frac{1}{2} \mathbf{n}_{\mathbf{2}}^{\mathbf{2}}-i_{\mathbf{2}}+\left(\mathbf{n}_{\mathbf{1}}-\mathbf{n}_{\mathbf{2}}\right) \cdot \boldsymbol{\sigma}\right)^{\mathbf{2}} \\
\left.\left(\frac{1}{2} \mathbf{n}_{\mathbf{3}}^{\mathbf{2}}+i_{\mathbf{3}}-\frac{\mathbf{1}}{\mathbf{2}} \mathbf{n}_{\mathbf{4}}^{\mathbf{2}}-i_{\mathbf{4}}+\left(\mathbf{n}_{\mathbf{3}}-\mathbf{n}_{\mathbf{4}}\right) \cdot \boldsymbol{\sigma}\right)^{\mathbf{2}}\right]=0 .
\end{gathered}
$$

The lowest order terms in (12), namely the coefficient of $t^{3+\sigma \cdot(4 / \sqrt{3},-2 / \sqrt{3},-2 / \sqrt{3})}$, gives a quartic relation which simplifies to the following quadratic one

$$
\begin{aligned}
& \left(\frac{2 \sigma_{1}-\sigma_{2}-\sigma_{3}}{\sqrt{3}}+1\right)^{2} B_{0}(\sigma) B_{0}\left(\sigma+\frac{1}{\sqrt{3}}(2,-1,-1)\right) \\
& =\left(\frac{\sigma_{2}-\sigma_{3}}{\sqrt{3}}\right)^{2}\left(\frac{\sigma_{1}+\sigma_{2}-2 \sigma_{3}}{\sqrt{3}}\right)^{2}\left(\frac{\sigma_{1}-2 \sigma_{2}+\sigma_{3}}{\sqrt{3}}\right)^{2} \\
& \quad \times\left(\frac{\sigma_{1}+\sigma_{2}-2 \sigma_{3}}{\sqrt{3}}+1\right)^{2}\left(\frac{\sigma_{1}-2 \sigma_{2}+\sigma_{3}}{\sqrt{3}}+1\right)^{2} \\
& \quad \times B_{0}\left(\sigma+\frac{1}{\sqrt{3}}(1,-2,1)\right) B_{0}\left(\sigma+\frac{1}{\sqrt{3}}(1,1,-2)\right) .
\end{aligned}
$$

By imposing (3), these are solved by

$$
\begin{aligned}
B_{0}^{\left[G_{2}\right]}(\boldsymbol{\sigma})= & \prod_{i<j}^{3} \frac{1}{G\left[1 \pm \frac{1}{\sqrt{3}}\left(\sigma_{i}-\sigma_{j}\right)\right]} \\
& \times \prod_{\substack{i j k \\
\text { cyclic }}}^{3} \frac{1}{G\left[1 \pm \frac{1}{\sqrt{3}}\left(2 \sigma_{i}-\sigma_{j}-\sigma_{k}\right)\right]} .
\end{aligned}
$$

The 1-instanton contribution is obtained by considering the coefficient of the next order $t^{3+\boldsymbol{\sigma} \cdot(\sqrt{3}, 0,-\sqrt{3})}$ term: all $B_{0}(\boldsymbol{\sigma})$ factors drop out and we obtain just

$$
\left.Z_{1}(\boldsymbol{\sigma})^{\left[G_{2}\right]}\right|_{\sigma_{3}=-\sigma_{1}-\sigma_{2}}=\frac{2}{3 \sigma_{1}^{2} \sigma_{2}^{2}\left(\sigma_{1}+\sigma_{2}\right)^{2}}
$$

in agreement with [28]. The next order in $t$ gives the 2-instanton term

$$
\left.Z_{2}(\boldsymbol{\sigma})^{\left[G_{2}\right]}\right|_{\sigma_{3}=-\sigma_{1}-\sigma_{2}}=\frac{3\left[9 \sigma_{1}^{4}\left(6 \sigma_{2}^{2}+1\right)+18 \sigma_{1}^{3}\left(6 \sigma_{2}^{3}+\sigma_{2}\right)+3 \sigma_{1}^{2}\left(18 \sigma_{2}^{4}+9 \sigma_{2}^{2}-2\right)+6 \sigma_{1} \sigma_{2}\left(3 \sigma_{2}^{2}-1\right)+\left(1-3 \sigma_{2}^{2}\right)^{2}\right]}{\sigma_{1}^{2}\left(1-3 \sigma_{1}^{2}\right)^{2} \sigma_{2}^{2}\left(1-3 \sigma_{2}^{2}\right)^{2}\left(\sigma_{1}+\sigma_{2}\right)^{2}\left[1-3\left(\sigma_{1}+\sigma_{2}\right)^{2}\right]^{2}}
$$


It should be possible to apply the approach proposed here to general class- $\mathcal{S}$ theories [29] by studying the related isomonodromic deformation problem (for example for linear and circular quivers). It would be also interesting to extend the analysis to non-self-dual $\Omega$ background, which should amount to quantum $\tau$ systems, and its lift to five dimensional gauge theories on $\mathbb{R}^{4} \times S^{1}$, which should correspond to $q$-difference $\tau$ systems [30-32]. Finally, it would be great to apply similar ideas to models with less or no supersymmetry. Let us outline some possible concrete research lines in this direction. The renormalization group evolution at strong coupling can be analyzed through late time expansion of the $\tau$ functions. In particular, in [33] the solution in this regime for the $A_{n}$ series has been given in terms of a matrix model describing the theory around the massless monopoles point. Confining $\mathcal{N}=1$ vacua can be studied through the addition of a mass term for the chiral supermultiplet [16]. The $\mathcal{N}=0$ case can be reached by giving a mass to its complex scalar field component only. The counterpart in the $\tau$ system and its matrix model solution of these mass deformations deserves to be studied in order to get an effective matrix model description of the confining string world sheet. A complementary approach would be the analysis of $\mathcal{N}=1$ breaking of theories in class $\mathcal{S}$ along the lines of [34]. More in general, the exploration of the renormalization group of gauge theories in the presence of surface defects, possibly in some special scaling regimes, might reveal interesting structures related to integrable systems and their deformations.

We would like to thank F. Del Monte, M. Mariño, and T. Nosaka for fruitful discussions. This research is partially supported by the Instituto Nazionale di Fisica Nucleare Research Projects Gauge and String Theory and String Theory and Fundamental Interactions, by Progetti di Rilevante Interesse Nazionale "Geometria delle varietà algebriche" and by PRIN "Non-Perturbative Aspects of Gauge Theories and Strings".

[1] G. 't Hooft, Nucl. Phys. B138, 1 (1978).

[2] N. Seiberg and E. Witten, Nucl. Phys. B431, 484 (1994).

[3] N. A. Nekrasov, arXiv:hep-th/0306211.

[4] S. Gukov and E. Witten, arXiv:hep-th/0612073.

[5] D. Gaiotto, A. Kapustin, N. Seiberg, and B. Willett, J. High Energy Phys. 02 (2015) 172.

[6] S. H. Katz, A. Klemm, and C. Vafa, Nucl. Phys. B497, 173 (1997).
[7] For an introduction to resurgence in QFT, see, for example, M. Marino, Instantons and Large N (Cambridge University Press, Cambridge, England, 2015).

[8] S. Cecotti and C. Vafa, Nucl. Phys. B367, 359 (1991).

[9] M. Jimbo, T. Miwa, and A. K. Ueno, Physica (Amsterdam) D2, 306 (1981).

[10] M. Jimbo, T. Miwa, and A. K. Ueno, Physica (Amsterdam) D2, 407 (1982).

[11] H. Nakajima and K. Yoshioka, Inventiones Mathematicae 162, 313 (2005).

[12] J. Kim, S.-S. Kim, K.-H. Lee, K. Lee, and J. Song, J. High Energy Phys. 11 (2019) 092; 06 (2020) 124(E).

[13] N. Nekrasov, arXiv:2007.03646.

[14] A. Gorsky, I. Krichever, A. Marshakov, A. Mironov, and A. Morozov, Phys. Lett. B 355, 466 (1995).

[15] E. J. Martinec and N. P. Warner, Nucl. Phys. B459, 97 (1996).

[16] N. Seiberg and E. Witten, Nucl. Phys. B426, 19 (1994); B430, 485 (1994).

[17] G. Bonelli, O. Lisovyy, K. Maruyoshi, A. Sciarappa, and A. Tanzini, Lett. Math. Phys. 107, 2359 (2017).

[18] G. Bonelli, F. Del Monte, P. Gavrylenko, and A. Tanzini, Commun. Math. Phys. 377, 1381 (2020).

[19] J. D. Edelstein, M. Marino, and J. Mas, Nucl. Phys. B541, 671 (1999).

[20] N. Bourbaki, in Lie Groups and Lie Algebras (SpringerVerlag Berlin Heidelberg, 2005), Chap. VIII, Sec. 7, https:// www.springer.com/gp/book/9783540688518.

[21] A. Its, O. Lisovyy, and Y. Tykhyy, Int. Math. Res. Not. 2015, 8903 (2015).

[22] A. Mironov and A. Morozov, Phys. Lett. B 773, 34 (2017).

[23] G. V. Dunne, J. Phys. A 52, 463001 (2019).

[24] Notice that if one chooses $\log \left(B_{0}\right) \sim \sum_{\mathbf{r} \in \mathbf{R}} c_{n, m}(\mathbf{r} \cdot \boldsymbol{\sigma})^{\mathbf{2 n}} \times$ $\log \left[(\mathbf{r} \cdot \boldsymbol{\sigma})^{2}\right]^{\mathbf{m}}$, then the equation itself dictates that $(n, m)=$ $(1,1)$ and $(2,0)$.

[25] M. A. Guest, A. R. Its, and C.-S. Lin, arXiv:1209.2045.

[26] N. Nekrasov and A. Okounkov, Progr. Math. 244, 525 (2006).

[27] M. Marino and N. Wyllard, J. High Energy Phys. 05 (2004) 021.

[28] C. A. Keller, N. Mekareeya, J. Song, and Y. Tachikawa, J. High Energy Phys. 03 (2012) 045.

[29] D. Gaiotto, J. High Energy Phys. 08 (2012) 034.

[30] G. Bonelli, A. Grassi, and A. Tanzini, Lett. Math. Phys. 109, 1961 (2019).

[31] M. Bershtein, P. Gavrylenko, and A. Marshakov, Theor. Math. Phys. 198, 157 (2019).

[32] G. Bonelli, F. Del Monte, and A. Tanzini, arXiv:2007.11596.

[33] G. Bonelli, A. Grassi, and A. Tanzini, Ann. Henri Poincaré 19, 743 (2018).

[34] G. Bonelli, S. Giacomelli, K. Maruyoshi, and A. Tanzini, J. High Energy Phys. 10 (2013) 227. 